\title{
Biexciton binding energy in fractional dimensional semiconductors
}

\author{
Troels F. Rønnow, ${ }^{1,2, *}$ Thomas G. Pedersen, ${ }^{1}$ and Bart Partoens ${ }^{3, \dagger}$ \\ ${ }^{1}$ Department of Physics and Nanotechnology, Aalborg University, Skjernvej 4A, DK-9220 Aalborg Øst, Denmark \\ ${ }^{2}$ ETH Zürich, Institut fur Theoretische Physik, Wolfgang-Pauli-Strasse 27, CH-8093 Zürich, Switzerland \\ ${ }^{3}$ Universiteit Antwerpen, Departement Fysica, Groenenborgerlaan 171, B-2020 Antwerpen, Belgium
}

(Received 23 August 2011; published 6 January 2012)

\begin{abstract}
Biexcitons in fractional dimensional spaces are studied using variational quantum Monte Carlo. We investigate the biexciton binding energy as a function of the electron-hole mass fraction $\sigma$ as well as study the dimensional dependence of biexcitons for $\sigma=0$ and $\sigma=1$. As our first application of this model we treat the $\mathrm{H}_{2}$ molecule in two and three dimensions. Next we investigate biexcitons in carbon nanotubes within the fractional dimensional model. To this end we find a relation between the nanotube radius and the effective dimension. The results of both applications are compared with results obtained using different models and we find a reasonable agreement. Within the fractional dimensional model we find that the biexciton binding energy in carbon nanotubes accurately scales as $E_{B}(r, \varepsilon)=1280 \mathrm{meV} \AA /(r \varepsilon)$, as a function of radius $r$ and the dielectric screening $\varepsilon$.
\end{abstract}

DOI: 10.1103/PhysRevB.85.045412

PACS number(s): 71.35.-y

\section{INTRODUCTION}

The confinement effect in nanostructures is responsible for pronounced binding energies of excitonic complexes. ${ }^{1-12}$ As a consequence charged excitons and biexcitons, which are not detectable in bulk materials at room temperature, play a significant role in highly confined systems such as quantum wells $\mathrm{s}^{2,4,5,13-15}$ and nanotubes. ${ }^{16-22} \mathrm{~A}$ popular method used to treat excitons and biexcitons in quasi-two-dimensional quantum wells is the fractional dimensional model, ${ }^{23,24}$ in which one models the confinement of the particles through a modified Laplace operator and integration measure. The fractional dimensional model was first applied to biexcitons by Singh et al. in Ref. 5. In their work they imposed a square structure on the wave function by which the Hamiltonian reduced to an excitonlike Hamiltonian. Later, this approach has been applied by other authors. ${ }^{4,25}$ The method by Singh et al. has the advantage that one arrives at an equation that can be solved analytically. However, the imposed square structure is an approximation and it would be desirable to solve the fractional dimensional biexciton equation without such a constraint. In this work we estimate a solution to the radial Hamiltonian in Hylleraas coordinates, where the only structural assumptions are given by the form of our variational trial function.

We have recently estimated the binding energy of trions in fractional dimensional spaces. ${ }^{26,27}$ In Ref. 27 we established the foundation for solving $N$ particle problems in fractional dimensional spaces using variational quantum Monte Carlo (VQMC). We used a model proposed by Palmer and Stavrinou ${ }^{24}$ to estimate the binding energy of the trion. Moreover, we demonstrated that the model produces results that are in good agreement with experiments for both carbon nanotubes (CNTs) and GaAs quantum wells. Thus the basis for solving the biexciton equation in fractional dimensional space has been established and additionally we expect that this model can predict the biexciton binding energy accurately for a wide variety of systems thereby uniting estimates for quantum wires, wells, and nanotubes in a single framework. ${ }^{1,4,5,23,25-29}$

In this paper we estimate the binding energy of biexcitons in fractional dimensional spaces. We present biexciton binding energies as a function of the fractional dimension $D$ as well as give the mass fraction spectrum for various dimensional parameters. Since the fractional dimensional model covers many different geometries we give two applications to illustrate the applicability. As the first application we estimate the energy of the hydrogen molecule. This application serves as an illustrative and simple example of how this model can be applied to systems of integer dimension. As the second application of this model we investigate biexcitons on the surface of a cylinder: First we establish a relationship between the cylinder radius $r$ and the fractional dimension $D$. To this end we use the model given in Refs. 18 and 20 to determine the exciton energy in CNTs. Then we calculate the biexciton energy as a function of the cylinder radius and the results are compared with the results given in Refs. 16, 17, 19, and 21. Finally we present the biexciton binding energies for various CNT species, before we conclude on the applicability of the fractional dimensional space technique.

\section{FRACTIONAL DIMENSIONAL MODEL FOR BIEXCITONS}

Following the approach in Ref. 27 we introduce three parameters $\alpha, \beta$, and $\gamma$ to control the collapse of axes and we define the fractional dimension as $D=\alpha+\beta+\gamma$. Moreover, we define the inner product as ${ }^{24,27,30}$

$$
\begin{aligned}
\left\langle\psi \mid \psi^{\prime}\right\rangle= & g^{N-1}(\alpha) g^{N-1}(\beta) g^{N-1}(\gamma) \int_{-\infty}^{\infty} \cdots \int_{-\infty}^{\infty} \\
& \times \psi^{*}\left(\vec{r}_{12}, \ldots, \vec{r}_{1 N}\right) \psi^{\prime}\left(\vec{r}_{12}, \ldots, \vec{r}_{1 N}\right) \\
& \times \prod_{i=2}^{N}\left|x_{1 i}\right|^{\alpha-1}\left|y_{1 i}\right|^{\beta-1}\left|z_{1 i}\right|^{\gamma-1} d x_{1 i} d y_{1 i} d z_{1 i},
\end{aligned}
$$

with

$$
g(\alpha)=\frac{\pi^{\alpha / 2}}{\Gamma\left(\frac{\alpha}{2}\right)},
$$

where $N$ is the number of particles and with $\Gamma(x)$ defined as

$$
\Gamma(x)=\int_{0}^{\infty} t^{x-1} e^{-t} d t .
$$


Adopting effective Rydbergs $\mathrm{Ry}^{*}=13.6 \mathrm{eV} \mu / \varepsilon^{2}$ and effective Bohr radii $a_{B}^{*}=0.529 \AA \varepsilon \mu$, as units of energy and length, the general $(m, n)$-Hamiltonian for $m$ electrons and $n$ holes can be approximated by introducing Hylleraas coordinates, removing the center-of-mass motion, and neglecting the contributions from the angular parts of the kinetic-energy operators. Here, $\mu=m_{e} m_{h} /\left(m_{e}+m_{h}\right)$ is the reduced electron-hole mass and $\varepsilon$ is the static dielectric constant. In the following, let $i, j$, and $k$ be electron indices, and $a, b$, and $c$ hole indices. Then after separating the kinetic energy into contributions from the electrons and holes we obtain ${ }^{31}$

$$
\begin{aligned}
\hat{H}= & -\sum_{i}\left(\frac{1}{1+\sigma} \hat{T}_{i}-\sum_{j>i} \frac{2}{r_{i j}}\right) \\
& -\sum_{a}\left(\frac{\sigma}{1+\sigma} \hat{T}_{a}-\sum_{b>a} \frac{2}{r_{a b}}\right)-\sum_{i, a} \frac{2}{r_{i a}},
\end{aligned}
$$

where $\sigma=m_{e} / m_{h}$ is the electron-hole mass fraction and

$$
\begin{aligned}
\hat{T}_{i}= & \sum_{j \neq i}\left(\frac{\partial^{2}}{\partial r_{i j}^{2}}+\frac{D-1}{r_{i j}} \frac{\partial}{\partial r_{i j}}\right)+2 \sum_{\substack{j<k \\
j, k \neq i}} \cos \left(\theta_{i j, i k}\right) \frac{\partial^{2}}{\partial r_{i j} \partial r_{i k}} \\
& +\sum_{a}\left(\frac{\partial^{2}}{\partial r_{i a}^{2}}+\frac{D-1}{r_{i a}} \frac{\partial}{\partial r_{i a}}\right)+2 \sum_{a<b} \cos \left(\theta_{i a, i b}\right) \frac{\partial^{2}}{\partial r_{i a} \partial r_{i b}},
\end{aligned}
$$

with

$$
\cos \left(\theta_{i a, i b}\right)=\frac{r_{i a}^{2}+r_{i b}^{2}-r_{a b}^{2}}{2 r_{i a} r_{i b}}
$$

Here $r_{i j}$ is the distance between the $i$ th and $j$ th particle (see Fig. 1). The kinetic-energy operators for the holes $\hat{T}_{a}$ are found by substituting $i, j, k$ with $a, b, c$ in $\hat{T}_{i}$.

In order to estimate the ground-state energy of Eq. (3) we chose a trial function

$$
\begin{aligned}
\Psi_{T}= & A\left(\psi_{\xi, \chi}+\psi_{\chi, \xi}\right) \Phi_{(\sigma+1) /(D-1), b_{1}, c_{1}} \\
& \times\left(r_{13}\right) \Phi_{(\sigma+1) /(D-1), b_{2}, c_{2}}\left(r_{24}\right),
\end{aligned}
$$

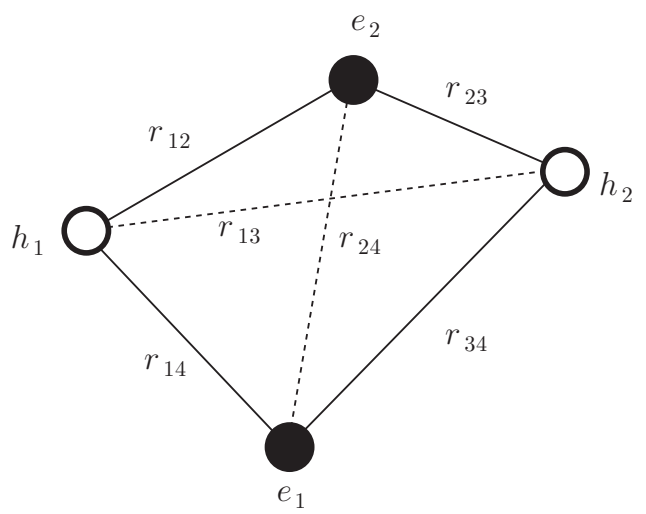

FIG. 1. Schematic illustration of the geometry of the biexciton. Filled circles illustrate electrons and hollow circles illustrate holes. Solid lines represent attractive Coulomb interactions while dashed lines are repulsive Coulomb interactions. with

$$
\psi_{\xi, \chi}=\exp \left[-\frac{2}{D-1}\left(\xi r_{12}+\chi r_{14}+\xi r_{34}+\chi r_{23}\right)\right],
$$

and

$$
\Phi_{a, b, c}(r)=\exp \left(\frac{a r}{1+b r+c r^{2}}\right),
$$

which has been constructed such that it can model two free excitons as well as two excitons bound to each other. Here all parameters $a, b_{1}, b_{2}, c_{1}, c_{2}, \chi, \xi>0$ are positive real numbers and $A$ is a normalization constant. The wave function is composed of three factors: $\psi_{\xi, \chi}+\psi_{\chi, \xi}, \Phi_{(\sigma+1) /(D-1), b_{1}, c_{1}}\left(r_{13}\right)$, and $\Phi_{(\sigma+1) /(D-1), b_{2}, c_{2}}\left(r_{24}\right)$. The first factor serves to model the attraction between the electrons and holes, the second factor models the electron-electron repulsion, and the third factor the hole-hole repulsion. When $\chi \rightarrow \xi$ the first factor reduces to $2 \psi_{\xi, \xi}$ thereby modeling a biexciton in which all electron-hole pairs are equally strongly bound. In contrast, when $\chi \rightarrow 0$ and $\xi>0$ one finds a superposition of free exciton pairs. The second and third factors of the wave function are modified Jastrow-type wave functions, which, in general, are known ${ }^{32}$ to model the correlation effects with good accuracy.

The local energy was found as ${ }^{33} E_{L}=\Psi_{T}^{-1} \hat{H} \Psi_{T}$ using the $(2,2)$ Hamiltonian defined in Eq. (3) for the probability distribution $\rho=\Psi_{T}^{2} W$ with

$$
W=\prod_{i=2}^{4}\left|x_{1 i}\right|^{\alpha-1}\left|y_{1 i}\right|^{\beta-1}\left|z_{1 i}\right|^{\gamma-1} .
$$

The expectation value of the energy can be evaluated as ${ }^{32}$

$$
E \approx \frac{1}{M} \sum_{i=1}^{M} E_{L}\left(x_{i}\right)
$$

where $M$ is the number of sampling points and $x_{i} \in \mathbb{R}^{3 N}$ is the $i$ th configuration sampled from the probability density $\rho(x)$. In the following we denote the biexciton energy $E$ and define the biexciton binding energy as $E_{B}=2 E_{X}-E$, which is positive for stable biexcitons. Here $E_{X}$ is the exciton binding energy. Also, we denote the singlet biexciton by $B_{\sigma}$ for mass fraction $\sigma$.

\section{RESULTS}

\section{A. General biexciton tendencies}

The variational coefficients in Eq. (5) were optimized using the stochastic gradient method. ${ }^{34,35}$ This method was used to optimize the energy and to determine the variational coefficients for selected mass fractions and dimensions. The coefficients in between the optimized ones were determined by using appropriate interpolations. We examined the autocorrelation function for a wide variety of configurations to ensure that the sampling points were uncorrelated. Typically simulations were carried out with acceptance rates between $70 \%$ and $90 \%$ and every walker was moved 50 steps in between sampling of the energy. With all calculations given here we provide the standard deviation as error bars. One should notice that while this quantity is a measure of the statistical uncertainty of the sampling process it does not include the 


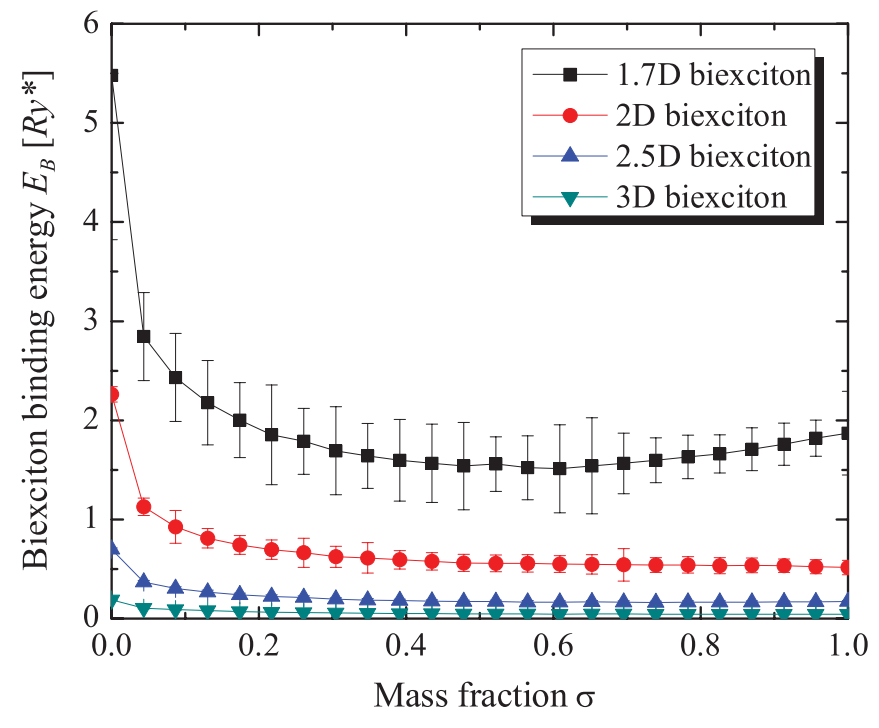

FIG. 2. (Color online) Mass fraction dependence of the fraction dimensional biexciton binding energy for $D=3, D=2.5, D=2$, and $D=1.7$. The dimensions were chosen such that the results cover biexcitons in bulk materials $(D=3)$, confined to the plane $(D=2)$, in quantum wells $(D=2.5)$, and in CNTs $(D=1.7)$. All energies are expressed in effective Rydberg Ry*, which is a material dependent unit. The results are therefore universal, rather than reflecting the properties of a specific material.

numerical inaccuracies arising from the singularities ${ }^{27}$ in the integration weight Eq. (6).

In Fig. 2 we have given the mass fraction dependence of the biexciton binding energy for $D=3, D=2.5, D=2$, and $D=1.7$. Results for $D=3$ and $D=2$ correspond to biexcitons in bulk materials and confined to a plane surface, respectively, whereas $D=2.5$ is expected to describe the properties of biexcitons in GaAs quantum wells of width ${ }^{27}$ $L \approx 240 \AA$. Finally, $D=1.7$ is expected to describe the properties of biexcitons in CNTs with an effective radius of $r=0.1 a_{B}^{*} \cdot{ }^{3,26,27,36}$ A special case of the three-dimensional biexciton is $B_{0}$, which includes the hydrogen molecule $\mathrm{H}_{2}$. We compared the results for $D=2$ and $D=3$ with the calculations done in Refs. 37 and 38, where the authors investigated the biexciton energy as a function of the electronhole mass fraction using the ordinary Laplace operators in two and three dimensions. Our model underestimated the energies with at most $6 \%$. We also found that the general tendencies of the mass fraction dependence are correct and thus our relatively simple estimate describes a wide variety of systems to reasonable precision.

In Fig. 2 it is observed that the mass fraction serves as a parameter to enhance the biexciton energy. For materials with $\sigma<0.1$ the biexciton binding energy increases significantly meaning that experimental observation of biexcitons will be more likely in nanostructures, where the electron-hole mass fraction is small. An example of a material where the mass fraction may play a role is $\mathrm{GaAs}$, with mass fractions around ${ }^{39}$ $\sigma=0.2$. In contrast, for CNTs where the mass fraction ${ }^{18,20}$ $\sigma \in[0.8,1]$, it is a reasonable approximation to assume that the dependence on $\sigma$ of the energy can be neglected. This simplifies the problem as one only needs to find the dependence of the energy on the effective cylinder radius. This allows for simple fits similar to those presented in Refs. 16 and 19. In terms of the mass fraction, CNTs are a poor choice of nanostructures for measuring biexciton binding energy. However, one should keep in mind that effective units play a significant role in the determination of the physical energies as well, and thus the energy dependence on the electron and hole masses does not solely enter through the mass fraction. The effective Rydberg is proportional to the reduced electron-hole mass $\mu=m_{e} m_{h} /\left(m_{e}+m_{h}\right)$. Therefore not only materials with low mass fraction $\sigma$ but also materials with large reduced masses $\mu$ are good candidates for measuring the biexciton binding energies. Finally, it is worth noting that the dielectric screening also plays an important role in determining the biexciton binding energies as the effective Rydberg is proportional to $\varepsilon^{-2}$. For CNTs this parameter is largely determined by the surrounding material and is typically taken to be $\mathrm{e}^{3,16-20,22,26,27} \varepsilon \in[3.4,4.5]$. Within this interval the effective Rydberg roughly varies by a factor of 2 and the relatively small screening, compared to, e.g., GaAs (Ref. 39) $(\varepsilon \approx 13)$, leads to a very large effective Rydberg Ry* in CNTs. The difference in dielectric constant yields energies differing by roughly a factor of 15 .

Next we focus on the biexcitonic energy as a function of dimensionality $D$, i.e., the confinement. The results are given in Fig. 3. First of all, the biexciton binding energy is increasing with decreasing dimension as would be expected from a physical point of view. Second, it is seen that the binding energy of the $B_{0}$ biexciton is larger than that of $B_{1}$ for all dimensions $D$. When $\sigma=0$ one has a case that is analogous with the hydrogen molecule $\mathrm{H}_{2}$ : In terms of the coordinates of relative motion it can be verified that the holes become localized when $\sigma$ is lowered by investigating the probability distribution $\rho$, similar to what was done for trions

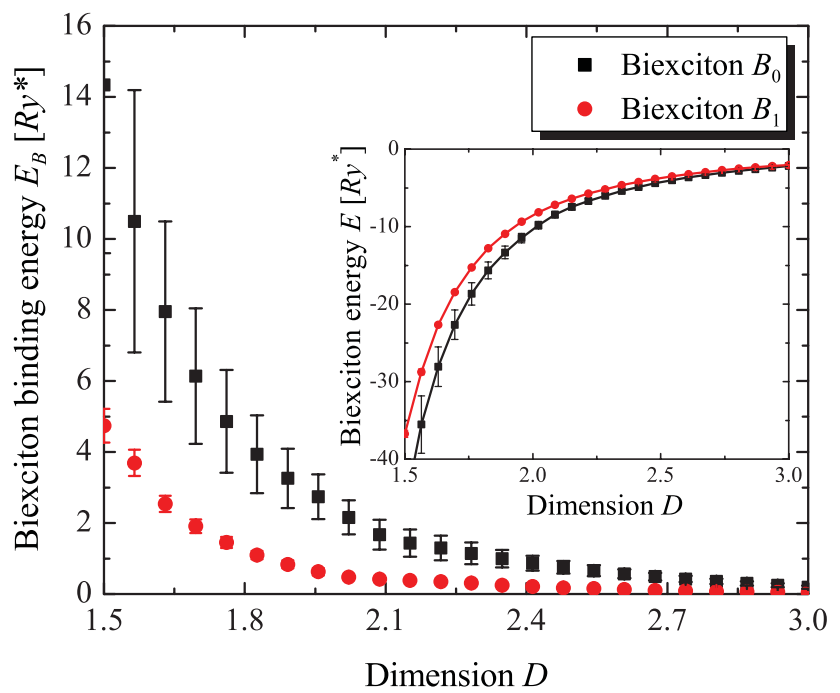

FIG. 3. (Color online) Biexcitonic energy for mass fractions $\sigma=$ 0 and $\sigma=1$ as a function of the dimension $D$. The dimension $D$ is a parameter that reflects the confinement of the biexciton. It is seen that increasing the dimensional confinement is an effective way of enhancing the biexciton binding energy as the energy is enhanced with more than a factor of 100 in going from $D=3$ to $D=1.5$ for $B_{1}$. Inset: Full energy $E$ of the $B_{0}$ and $B_{1}$ biexcitons. 
in Ref. 27. Consequently the kinetic energy is reduced as a result of the two holes (or electrons) becoming stationary, meaning that the binding energy $E_{B}$ is increased. In this context it is important to note that the exciton Hamiltonian is independent of the mass fraction ${ }^{1}$ and that this is not the case for the general many body fractional dimensional Hamiltonian in Eq. (3). If one calculates the fraction between the binding energy of $B_{0}$ and $B_{1}$ it is found that the $B_{0}$ binds from 2.5 times stronger than $B_{1}$ at $D=1.5$ up to 4 times at $D=3$. On the one hand this clearly demonstrates that the choice of material (which determines the mass fraction) is not negligible when designing nanostructures in which biexcitons should be observable. On the other hand, however, the confinement constitutes a much more effective parameter in enhancing the biexciton binding energy as one roughly finds a factor of 100 in difference between the energies at $D=3$ and $D=1.5$ for $B_{1}$. In conclusion, four key parameters determine the binding energy of the biexciton, the first being the electron-hole mass fraction, the second being the reduced electron-hole mass, the third being the dielectric screening, and the fourth being the confinement of the particles. The first three are determined by the choice of material and suspension medium whereas the fourth is a result of the system geometry. Especially, low-dimensional nanostructures with $D<2.0$ and low dielectric constants are promising to measure the biexciton binding energy at room temperature.

All binding energies presented up to now have been given in units of effective Rydberg Ry* and are therefore universal energies. This means that they can be applied to a wide variety of systems if one knows the electron and hole masses, the static dielectric constant and the dimension of the system. Biexcitons form a quite general class of complexes covering everything from the hydrogen molecule $\mathrm{e}^{40-42}$ to the positronium molecule. ${ }^{43}$ Besides covering the standard two-dimensional (2D) and 3D cases, the interpolation of the Laplacian operators makes the model applicable in the description of biexciton energies in quasi-two-dimensional quantum wells ${ }^{4,25}$ and CNTs. ${ }^{26,27,36}$ In the hope that our results will serve as a guideline for experimentalists as well as other theoretical physicists we here give curve fits of the energy as a function of the dimension $D$ for the two limiting cases ( $\sigma=0$ and $\sigma=1)$,

$$
E_{\sigma}(D)=\sum_{i=1}^{5} \frac{c_{i}}{D^{i-1}} e^{-D}
$$

with the coefficients given in Table I.

To demonstrate how this work is applied to real physical systems we give two examples of applications. First, we study the $\mathrm{H}_{2}$ molecule in two and three dimensions. This example serves to confirm that the model exhibits the correct limiting behavior. Then, in the next section, we turn our attention

TABLE I. Fitting coefficients in units of $R y^{*}$ for biexciton binding energies.

\begin{tabular}{cccccc}
\hline \hline & $c_{1}$ & $c_{2}$ & $c_{3}$ & $c_{4}$ & $c_{5}$ \\
\hline$B_{0}$ & -400 & 3361 & -11728 & 18359 & -11495 \\
$B_{1}$ & -308 & 2468 & -8489 & 13251 & -8379 \\
\hline \hline
\end{tabular}

toward a more sophisticated application, namely biexcitons in CNTs.

\section{B. $\mathrm{H}_{2}$ molecule}

For the $\mathrm{H}_{2}$ molecule the effective Rydberg is $\mathrm{Ry}^{*}=$ $13.6 \mathrm{eV}$ and the electron-hole mass fraction is $\sigma=0$. Using Eq. (8) we find $E_{0}(3)=-2.23 \mathrm{Ry}^{*}=-30.32 \mathrm{eV}$, in three dimensions, which should be compared with the true energy ${ }^{40}$ of $-31.66 \mathrm{eV}$, giving an error of $4.2 \%$. Similarly, we can now estimate the energy of the $\mathrm{H}_{2}$ molecule in two dimensions as $E_{0}(2)=-10.16 \mathrm{Ry}^{*}=-138 \mathrm{eV}$. This result should be compared with the variational result reported in Ref. 42 where the authors found $E=-141 \mathrm{eV}$, giving a difference of roughly $2.5 \%$.

Since the description of atoms and molecules in Hylleraas coordinates has proven to be precise in comparison with experiment $^{31}$ we expect that the deviation arises from the variational trial wave function. While modification of the trial wave function would be likely to give more precise energies, it would, however, be preferable to extend this model using a diffusion quantum Monte Carlo (DQMC) approach, in which case the obtained energies would be exact within the obtained statistical errors. ${ }^{32}$

\section{BIEXCITONS IN CARBON NANOTUBES}

As the second application we study biexcitons on the surface of a cylinder. While the cylindrical geometry has been used successfully in the description of electron-hole complexes in CNTs, ${ }^{3,16,18-20}$ biexcitons in CNTs have not been studied using a fractional dimensional model yet.

This section is organised as follows: First we give a brief review of past efforts with regard to the biexciton binding energy in CNTs followed by a discussion of the effective units in CNTs, which is important in order to conclude on the agreement between the models for actual CNTs. Then we discuss the dimensionality of CNTs, i.e., the relation between the nanotube radius $r$ and the dimension $D$, for which we establish an expression. Using this relation we estimate the biexciton binding energy in CNTs as function of nanotube radius. These results are compared with the results in Refs. 16, 17,19 , and 21 and we conclude on the overall agreement with these studies. We further explain the Kataura modulation of the biexciton binding energies. Through these considerations we show that the biexciton binding energy is proportional to $r^{-1} \varepsilon^{-1}$. Finally, we calculate the biexciton binding energy for a wide variety of CNT species.

\section{A. Brief review of past CNT biexciton efforts}

During the last six years, many papers have investigated biexcitons and nonlinear optical properties of CNTs. ${ }^{16,17,19,21,44-51}$ Biexcitons in CNTs were first studied ${ }^{16}$ by Pedersen et al. in 2005. In this work the authors used a cylindrical geometry and assumed that the biexciton was completely delocalized around the circumference to arrive at an effective one-dimensional Hamiltonian. The problem was solved using a Gaussian basis expansion and it was concluded that biexcitons in CNTs are stable particles. Later, Kammerlander et al. investigated the full cylindrical 
biexcitonic Hamiltonian through VQMC ${ }^{17,19}$ and DQMC. ${ }^{19}$ The authors concluded that the biexciton binding energy was twice the magnitude of that found in Ref. 16. Moreover, they found that the biexciton binding energies exhibit a pronounced Kataura behavior in contrast to the findings in Ref. 16 where it was concluded that the biexciton binding energy to a large extent only depends on the radius of the CNTs rather than the chiral index. Recently a one-dimensional investigation ${ }^{21}$ of biexcitons was carried out in which the author concluded that the biexciton binding energy scales as $r^{-0.6}$. This is in contrast to the $r^{-1}$ dependence predicted in Ref. 16. Finally, the first study of dependence on the dielectric screening was done in Ref. 47. It was found that the screening is more pronounced for biexcitons than for excitons in large diameter CNTs meaning that the biexciton binding energy is expected to be less than half the values predicted by phenomenological models.

In 2007 the first experimental study reporting on biexcitons in $\mathrm{CNTs}^{48}$ was conducted by Styers-Barnett et al. Studying the transient absorption spectra of SWCNTs using a femtosecond laser pulse they observed a shift in the induced absorption bands, which was attributed to biexciton formation. The observed binding energies were found to be in good agreement with theoretical estimates. ${ }^{16,17,19}$ As of today no signatures of the biexciton have been found in photoluminescence spectra. ${ }^{44}$ It has been suggested that multiple excitons recombine through a nonradiative Auger process. ${ }^{44}$ However, this idea was investigated theoretically by Watanabe et al. and it was concluded that biexciton formation is not prevented by Auger recombination. ${ }^{46}$ While biexcitons have not been observed directly in photoluminescence spectra ${ }^{44}$ it is still possible that biexcitons will be observed through two-photon absorption experiments. But in light of the many different results presented in literature on biexcitons in CNTs, there is need for further investigations of the biexciton binding energies.

The goal of the following subsections is to show how one can apply the fractional dimensional model to estimate the biexciton energy in highly confined systems. CNTs are especially well suited for this purpose due to their lowdimensional geometry. As the fractional dimensional model has previously been applied successfully to excitons and trions in CNTs, we also expect that it will predict the biexciton binding energies accurately.

\section{B. Effective units of CNTs}

All energies in Sec. II are expressed in effective Rydberg $\mathrm{Ry}^{*}$ and are therefore not applicable when comparing with experiment. For completeness of this work we give an example of how to convert the effective energies into real physical values. We consider CNTs with chirality $(7,5)$ as an example. In our previous work we demonstrated that a dielectric constant of $\varepsilon=3.4$ predicts the trion energy with high precision when compared with the experimental results in Ref. 52. With this in mind we assume that $\varepsilon=3.4$ is a reasonable value. In order to obtain the effective electron and hole masses we used a nearest-neighbor tight-binding model with a transfer integral of $^{50} t=-2.89 \mathrm{eV}$ and a hopping integral of $s=0.1$. Using this model the effective electron and hole masses for $(7,5)$ CNTs are found to be $m_{e} \approx 0.081 m_{0}$ and $m_{h} \approx 0.087 m_{0}$, respectively, giving a reduced mass of $\mu \approx 0.042 m_{0}$ resulting in an effective Rydberg ${ }^{27} \mathrm{Ry}^{*} \approx 49 \mathrm{meV}$. Using that $(7,5)$ CNTs have an effective Bohr radius of $a_{B}^{*}=0.529 \AA \varepsilon / \mu=$ $43 \AA$, the effective radius of a $(7,5) \mathrm{CNT}$ is $r=0.092 a_{B}^{*}$. In order to determine the dimension of the cylinder we followed the approach given in Ref. 36, which has been proven to be quite accurate. ${ }^{26}$ The dimension was determined as follows: One calculates the exciton energy $E_{X}(r)$ on the surface of a cylinder. The dimension $D$ is then given by the relation $^{36} D=\sqrt{-4 / E_{X}}+1$. This method ensures that the exciton binding energy is predicted correctly by the fractional dimensional model. For $(7,5)$ CNTs one finds an effective dimension of $D=1.70$ for which the binding energy is $2 E_{X}-E_{1} \approx 1.88 \mathrm{Ry}^{*}=92 \mathrm{meV}$.

For actual CNTs the effective radius (i.e., in units of $a_{B}^{*}$ ) is found in a small range around $0.1 a_{B}^{*}$, which can easily be verified either by using same the tight-binding approach or by using the expressions for the effective electron and hole masses given in Ref. 53. The latter was used in Ref. 19 to determine the effective units, whereas the former has been used in Ref. 20. Within the model used by Kammerlander $97 \%$ of the semiconducting nanotubes with chiral index $10 \leqslant 2 n+$ $m \leqslant 200$ have an effective Bohr radius $0.08 a_{B}^{*}<r<0.15 a_{B}^{*}$. Within the tight-binding model it is found that $98 \%$ are in the region $0.07 a_{B}^{*}<r<0.13 a_{B}^{*}$.

\section{Dimensionality of CNTs}

We found the exciton energy (in effective Ry*) on the surface of a cylinder using a basis expansion as explained in Ref. 20 for $r \in[0,0.4] a_{B}^{*}$. Using the exciton energy the dimension was found as explained in Sec. IV B. The result has been shown in the inset of Fig. 4. The dimension is accurately fitted by

$$
D(r) \approx 2-\exp \left(-a_{1} r^{b_{1}}\right)+r \exp \left(-a_{2} r^{b_{2}}\right),
$$

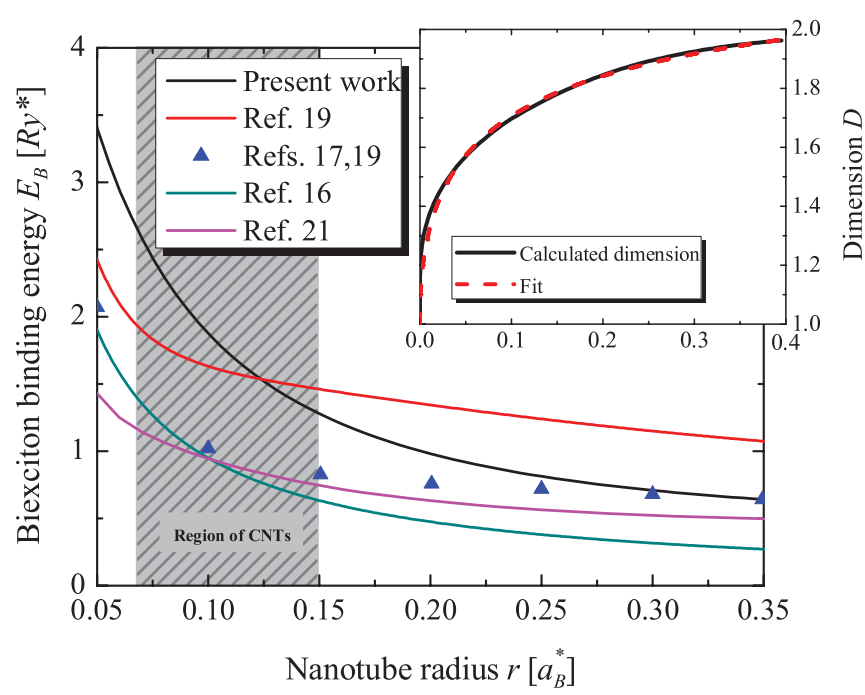

FIG. 4. (Color online) Binding energy as function of $r$ where the mapping in Eq. (9) is used to convert the radius into dimension. For comparison we have also given the results in Refs. 16, 17, 19, and 21. Inset: Relation between the effective nanotube radius and the dimension $D$ calculated using the model in Ref. 20. The fit is given in Eq. (9). 
with $a_{1}=3.4, b_{1}=0.48, a_{2}=2.0$, and $b_{2}=0.23$. Notice that the limits of $D(r)$ are intuitive: for $r \rightarrow 0$ the system effectively becomes a one-dimensional system as $D(r) \rightarrow 1$ and for $r \rightarrow \infty$ the dimension is found to be $D(r) \rightarrow 2$. Using this relation between $r$ and $D$ we calculated the biexciton binding energy $E_{B}(D)$ as a function of $r$, which is shown in Fig. 4, together with other results published in literature.

First of all, notice that the overall agreement between the results presented here, the basis expansion presented in Ref. 16, the VQMC calculations given in Ref. 17, the DQMC curve fit given in Ref. 19 and the one-dimensional result given in Ref. 21 is reasonably good. The model presented here gives binding energies that are well above the one-dimensional models, Refs. 16 and 21, and that are higher than or equal to the VQMC estimate in Ref. 17 on the interval [0.05,0.35] $a_{B}^{*}$.

Second, the difference in the asymptotic behavior of the results presented here and the DQMC results in Ref. 19 when $r \rightarrow \infty$ is noticed. The small deviation is a result of the quality of the variational estimate in Eq. (8), which predicts a binding energy of $0.58 R y^{*}$, in two dimensions, whereas the correct value for $\sigma=1$ is $^{37} 0.77 \mathrm{Ry}^{*}$. Improvement of the trial function would presumably correct this problem. For the purpose of studying biexcitons in CNTs the limiting behavior is, however, of little importance as the effective radii are found on the interval $0.07 a_{B}^{*}<r<0.15 a_{B}^{*}$, as discussed in the previous section. This area has been shaded in Fig. 4. Finally, one notices that for radii $r$ below $0.12 a_{B}^{*}$ the fractional dimensional model predicts a larger energy than previous investigations.

\section{Scaling of biexciton binding energies in CNTs}

The slope with respect to radius of the biexciton binding energy in effective units is important as it determines properties of the energy spectrum that may be helpful in identifying the biexciton formation. For excitonic complexes in CNTs the Kataura modulation in the energy spectrum arises solely from the effective units $R y^{*}=13.6 \mathrm{eV} \mu / \varepsilon^{2}$ and $a_{B}^{*}=0.529 \AA \varepsilon / \mu$ through the effective masses. In fact, one gets a very pronounced Kataura plot if the biexciton binding energy were constant $E_{B}=c_{0}$ as a function of the radius in effective Bohr radii $a_{B}^{*}$. In this case the biexciton binding energy becomes proportional to the reduced electron-hole mass $\mu$, which exhibit this pattern. ${ }^{10,53}$ For $E_{B}=c_{n} r^{n}$ (in Ry*) one finds that $E_{B}$ (in $\mathrm{eV}$ ) is proportional to $\mu^{n+1}$. If, however, the energy is well described by $c_{-1} / r$ it is evident that the Kataura structure vanishes as

$$
\begin{aligned}
E_{B}(r) & =\frac{c_{-1}}{r\left[a_{B}^{*}\right]}\left[\mathrm{Ry}^{*}\right]=\frac{c_{-1} a_{B}^{*}}{r[\AA]}\left[\mathrm{Ry}^{*}\right] \\
& =\frac{c_{-1} a_{B}^{*}}{r[\AA]} \mathrm{Ry}^{*}[\mathrm{eV}]=7.19 \AA \mathrm{eV} \frac{c_{-1}}{\varepsilon r[\AA]}[\mathrm{eV}],
\end{aligned}
$$

becomes independent of $\mu$. This was already noted in Ref. 16 . Here square brackets have been used to indicate the units used. In our case $E_{B} \propto r^{-1}$ for $r \in\left[0.05 a_{B}^{*}, 0.15 a_{B}^{*}\right]$ and $c_{-1}$ was found to be $c_{-1} \approx 0.178 \mathrm{Ry}^{*} a_{B}^{*}$ giving $E_{B}(r, \varepsilon) \approx$ $1280 \mathrm{meV} \AA /(r \varepsilon)$. Thus as a result one would expect that the actual CNT biexciton binding energies, to a large extent, only depend on the CNT radius and not on the chiral index.

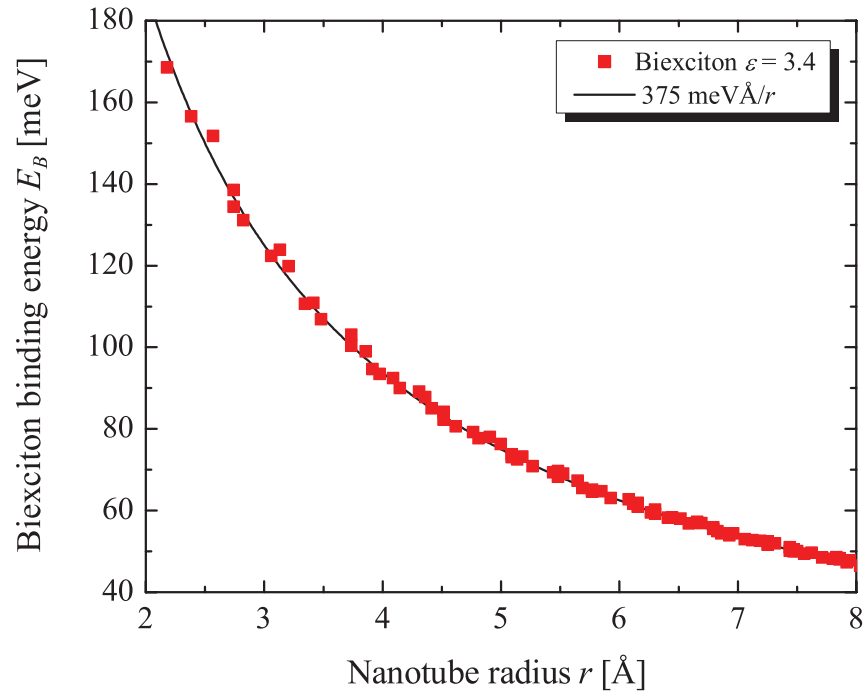

FIG. 5. (Color online) Biexciton binding energy in CNTs calculated using the fractional dimensional model (red squares). The results were obtained by estimating the effective CNT radius for the individual CNTs using a nearest-neighbor tight-binding model. The radius was converted into an effective dimension through Eq. (9) from which the biexciton binding energy was found using Eq. (8). Finally the energy was converted into physical units using the definition of the effective Rydberg. The solid line is a fit of found energies. It is seen that the CNT binding energy within this model follows roughly the relation $E_{B}(r)=375 \mathrm{meV} \AA / r$.

Moreover, the scaling of the biexciton binding energy in terms of the dielectric constant also simplifies as the binding energy becomes proportional to $\varepsilon^{-1}$.

We calculated the actual CNT binding energy for a wide range of CNTs as explained in Sec. IV B using $\varepsilon=3.4$. The results are shown in Fig. 5. In comparison with the results in Ref. 19 the Kataura effect is much more subtle in the present calculations as was also expected from the previous discussion. Consequently the energies are well described by the simple relation $E_{B}(r) \approx 375 \mathrm{meV} \AA / r$, which is in good agreement with what was found in Ref. 16, but in contrast to the $r^{-0.6}$ dependence found in Ref. 21 . We also found the actual binding energies to be twice the magnitude of those in Refs. 16 and 21, while only slightly higher than those reported in Ref. 19.

In Ref. 54 the exciton binding energy was found to scale as $\varepsilon^{-1.6}$ with the dielectric constant and the trion binding energy has been found to scale as $\varepsilon^{-1.56}$ in Ref. 20. As discussed earlier we here find that the biexciton binding energy scales as $\varepsilon^{-1}$, which is in good agreement with a recent calculation that include some many-body effects but excludes the screening of interaction. ${ }^{47}$ It is worth noting, however, that the present model produces energies of the same magnitude as other phenomenological models, ${ }^{47}$ meaning that (1) the results presented here are likely to be an overestimate of what will be found experimentally, and (2) that the present work does not account the screening of the interaction and thus we find no saturation of the binding energy as a function of the dielectric constant, in contrast to the what was found in Ref. 47 for the calculation including screening effects. 


\section{SUMMARY}

In this paper we have shown how to apply VQMC to estimate the biexciton binding energy in fractional dimensional semiconductor structures. We have demonstrated the applicability of the method by calculating the binding energy of the $\mathrm{H}_{2}$ molecule in two and three dimensions as well as estimating the binding energy of biexcitons in CNTs. In both cases we have found a reasonable agreement with other theoretical work. For CNTs we have established a relation between the fractional dimension and the tube radius, and we have found that the biexciton binding energy is inversely proportional to the radius and the dielectric constant. Consequently we predict that the Kataura modulation of the biexciton binding energy is less pronounced than what was found by Kammerlander et al. ${ }^{19}$ Finally we find the biexciton binding energy in CNTs scales accurately as $E_{B}(r, \varepsilon)=1280 \mathrm{meV} \AA /(r \varepsilon)$ within the fractional dimensional model. *tfr@ nanophysics.dk; http://www.nanophysics.dk

†bart.partoens@ua.ac.be

${ }^{1}$ X. F. He, Phys. Rev. B 43, 2063 (1991).

${ }^{2}$ A. Thilagam, Phys. Rev. B 56, 4665 (1997).

${ }^{3}$ T. G. Pedersen, Phys. Rev. B 67, 073401 (2003).

${ }^{4}$ Z. P. Wang and X. X. Liang, Solid State Commun. 150, 356 (2010).

${ }^{5}$ J. Singh, D. Birkedal, V. G. Lyssenko, and J. M. Hvam, Phys. Rev. B 53, 15909 (1996).

${ }^{6}$ P. K. Basu, Theory of Optical Processes in Semiconductors (Oxford Science Publications, New York, 2005).

${ }^{7}$ G. Bastard, E. E. Mendez, L. L. Chang, and L. Esaki, Phys. Rev. B 26, 1974 (1982).

${ }^{8}$ T. Ando, J. Phys. Soc. Jpn. 66, 1066 (1997).

${ }^{9}$ C. D. Spataru, S. Ismail-Beigi, L. X. Benedict, and S. G. Louie, Phys. Rev. Lett. 92, 077402 (2004).

${ }^{10}$ T. G. Pedersen, Carbon 42, 1007 (2004).

${ }^{11}$ J. R. Guest, T. H. Stievater, X. Li, J. Cheng, D. G. Steel, D. Gammon, D. S. Katzer, D. Park, C. Ell, A. Thränhardt, G. Khitrova, and H. M. Gibbs, Phys. Rev. B 65, 241310 (2002).

${ }^{12}$ F. Wang, G. Dukovic, L. E. Brus, and T. F. Heinz, Science 308, 838 (2005).

${ }^{13}$ A. Matos-Abiague, J. Phys.: Condens. Matter 14, 4543 (2002).

${ }^{14}$ R. Escorcia, J. Sierraortega, I. Mikhailov, and F. Betancur, Physica B 355, 255 (2005).

${ }^{15}$ L. de Dios, A. B. Alfonso, A. M. Abiague, and L. E. Oliveira, J. Appl. Phys. 82, 3155 (1997).

${ }^{16}$ T. G. Pedersen, K. Pedersen, H. D. Cornean, and P. Duclos, Nano Lett. 5, 291 (2005).

${ }^{17}$ D. Kammerlander, D. Prezzi, G. Goldoni, E. Molinari, and U. Hohenester, Physica E 40, 1997 (2008).

${ }^{18}$ T. F. Rønnow, T. G. Pedersen, and H. D. Cornean, Phys. Lett. A 373, 1478 (2009).

${ }^{19}$ D. Kammerlander, D. Prezzi, G. Goldoni, E. Molinari, and U. Hohenester, Phys. Rev. Lett. 99, 126806 (2007).

${ }^{20}$ T. F. Rønnow, T. G. Pedersen, and H. D. Cornean, Phys. Rev. B 81, 205446 (2010).

${ }^{21}$ I. V. Bondarev, Phys. Rev. B 83, 153409 (2011).

${ }^{22}$ T. F. Rønnow, T. G. Pedersen, and H. D. Cornean, Physica E, doi:10.1016/j.physe.2011.04.001.

${ }^{23}$ D. R. Herrick and F. H. Stillinger, Phys. Rev. A 11, 42 (1975).

${ }^{24}$ C. Palmer and P. N. Stavrinou, J. Phys. A 37, 6987 (2004).

${ }^{25}$ I.-K. Oh and J. Singh, Phys. Rev. B 60, 2528 (1999).

${ }^{26}$ T. F. Rønnow, T. G. Pedersen, and H. D. Cornean, J. Phys. A 43, 474031 (2010).

${ }^{27}$ T. F. Rønnow, T. G. Pedersen, B. Partoens, and K. K. Berthelsen, Phys. Rev. B 84, 035316 (2011).

${ }^{28}$ H. Mathieu, P. Lefebvre, and P. Christol, Phys. Rev. B 46, 4092 (1992).
${ }^{29}$ K. F. Karlsson, M.-A. Dupertuis, H. Weman, and E. Kapon, Phys. Rev. B 70, 153306 (2004).

${ }^{30}$ M. Sadallah and S. I. Muslih, Int. J. Theor. Phys. 48, 3312 (2009).

${ }^{31}$ M. B. Ruiz, Int. J. Quantum Chem. 101, 261 (2005).

${ }^{32}$ J. Thijssen, Computational Physics (Cambridge University Press, Cambridge, England, 2007).

${ }^{33}$ The local energy contains many terms and no simple form has been found for which reason it has not been included here.

${ }^{34}$ A. Harju, B. Barbiellini, S. Siljamäki, R. M. Nieminen, and G. Ortiz, Phys. Rev. Lett. 79, 1173 (1997).

${ }^{35}$ X. Lin, H. Zhang, and A. M. Rappe, J. Chem. Phys. 112, 2650 (2000).

${ }^{36}$ T. G. Pedersen, Solid State Commun. 141, 569 (2007).

${ }^{37}$ J. Usukura, Y. Suzuki, and K. Varga, Phys. Rev. B 59, 5652 (1999).

${ }^{38}$ D. A. Kleinman, Phys. Rev. B 28, 871 (1983).

${ }^{39}$ A. V. Filinov, C. Riva, F. M. Peeters, Yu. E. Lozovik, and M. Bonitz, Phys. Rev. B 70, 035323 (2004).

${ }^{40}$ D. B. Kinghorn and L. Adamowicz, J. Chem. Phys. 113, 4203 (2000).

${ }^{41}$ W. K. Os and L. Wolniewicz, J. Chem. Phys. 41, 3663 (1964).

${ }^{42}$ J.-L. Zhu, X. Chen, and J.-J. Xiong, J. Phys.: Condens. Matter 3, 9559 (1991).

${ }^{43}$ P. M. Kozlowski and L. Adamowicz, Phys. Rev. A 48, 1903 (1993).

${ }^{44}$ K. Matsuda, T. Inoue, Y. Murakami, S. Maruyama, and Y. Kanemitsu, Phys. Rev. B 77, 033406 (2008).

${ }^{45}$ K. Watanabe, K. Asano, and T. Ogawa, J. Phys. Conf. Ser. 148, 012053 (2009).

${ }^{46}$ K. Watanabe, K. Asano, and T. Ogawa, Phys. Status Solidi C 6, 300 (2009).

${ }^{47}$ K. Watanabe and K. Asano, Phys. Rev. B 83, 115406 (2011).

${ }^{48}$ D. J. Styers-Barnett, S. P. Ellison, B. P. Mehl, B. C. Westlake, R. L. House, C. Park, K. E. Wise, and J. M. Papanikolas, J. Phys. Chem. C 112, 4507 (2008).

${ }^{49}$ L. Lüer, J. Crochet, T. Hertel, G. Cerullo, and G. Lanzani, ACS Nano 4, 4265 (2010).

${ }^{50}$ T. G. Pedersen and K. Pedersen, Phys. Rev. B 79, 035422 (2009).

${ }^{51}$ S. Uryu, H. Ajiki, and T. Ando, Phys. Rev. B 78, 115414 (2008).

${ }^{52}$ R. Matsunaga, K. Matsuda, and Y. Kanemitsu, Phys. Rev. Lett. 106, 037404 (2011)

${ }^{53}$ A. Jorio, C. Fantini, M. A. Pimenta, R. B. Capaz, Ge. G. Samsonidze, G. Dresselhaus, M. S. Dresselhaus, J. Jiang, N. Kobayashi, A. Grüneis, and R. Saito, Phys. Rev. B 71, 075401 (2005).

${ }^{54}$ V. Perebeinos, J. Tersoff, and P. Avouris, Phys. Rev. Lett. 92, 257402 (2004). 